\title{
Focus on Harsh Environment Mass Spectrometry
}

$\mathrm{T}$ The increasing trend toward miniaturization has resulted in most of the common forms of mass spectrometers becoming available in miniature and/or microengineered versions. Miniature versions of quadrupole, time-of-flight, magnetic sector, and various types of ion trap mass spectrometers have all been realized and mass spectra obtained. This has led to a number of field-portable instruments that are finding increasing application in environments previously thought impossible for locating a mass spectrometer. The selection of papers in this special issue arose from the Harsh Environment Mass Spectrometry (HEMS) workshop held in Ocean Beach, Florida, in September 2007. The HEMS workshop organizers are grateful to the editors of JASMS for this opportunity to report on recent work and draw the attention of the wider MS community to some of the exciting advances in HEMS.

But what exactly is a "harsh environment?" A useful working definition is "anywhere outside of the laboratory." This includes diverse scenarios such as airports, freshwater lakes, active volcanoes, deep ocean, interplanetary space, and the battlefield. The mass spectrometers discussed in this issue have been used in all of these environments. In June this year, the Phoenix spacecraft landed on the surface of Mars to conduct six experiments to study the history of water on that planet and to search for organic molecules in the subsurface soil of the Martian arctic region. One of the instruments on board the Phoenix is the thermal evolved gas analyzer, TEGA, incorporating a miniature magnetic sector mass spectrometer. The opening article in this special issue is from the laboratory of John Hoffman (University of Texas at Dallas), who is a pioneer in spacecraftborne MS instruments. A different (and novel) type of mass spectrometer is described in the second article by Herrero and co-workers from the NASA Goddard Space Flight Center. In their GEMS system, the integral of the time-of-flight is obtained and the TOF spectrum follows from the derivative of the raw data.

The three sequel articles, although describing Earthbased instruments, nevertheless involve harsh and chal-

Cover. Main image: Solstice on Mars. This image was acquired by NASA's Phoenix Mars Lander's Surface Stereo Imager (SSI) in the late afternoon of the 30th Martian day of the mission, June 25, 2008, hours after the beginning of Martian northern summer. SSI used its natural-color filters; therefore, the color of the image is as you would see on Mars. The image shows shadows from the SSI (left) and from the meteorological station mast (right) stretching toward the east as the sun dropped low in the west. Inset: The robotic arm scoop containing a Martian soil sample poised over the partially open door of the thermal and evolved gas analyzer (TEGA) oven to be heated prior to analysis via the Phoenix on board mass spectrometer. For a description of the TEGA see article by J. Hoffman et al., page 1377. lenging environments. Schlüter and Gentz (AlfredWegener Institute, Germany) present results of online $\mathrm{CH}_{4}$ and $\mathrm{CO}_{2}$ measurements in the surface $(5 \mathrm{~m})$ and bottom waters $(80 \mathrm{~m})$ of the Baltic Sea and in Lake Constance, respectively, using membrane inlet mass spectrometry (MIMS). A key issue is calibration and, in this case, a simple volumetric calibration methodology was used. Hemond et al. (MIT) report on the use of two MS instruments for the measurement of volatile organic compounds in natural waters. KOALA is a backpackable mass spectrometer operated from above the water surface, and NEREUS is an underwater instrument hosted in an autonomous underwater vehicle (AUV) linked to a communications network to provide chemical data in real time. Spatial profiling and sub-ppm detection limits are demonstrated with order of magnitude time savings compared to conventional analyses. 3D mapping and profiling of hazardous volcanic gas emissions using an in situ mass spectrometer are believed to be key steps toward a better understanding of the precursor geophysical phenomena associated with an eruption. Griffin and co-workers (Kennedy Space Center) with Diaz and colleagues (PRIAS-CENAT and the University of Costa Rica) report on an aircraft-based portable TOF MS system capable of monitoring atmospheric gases from sea level to 44,000 feet $(13.4 \mathrm{~km})$.

The final four papers in the current issue are concerned with ion trap MS technology. Wells et al. (Griffin Analytical) demonstrate a mobile MS instrument with an atmospheric pressure interface (API MS). The instrument (weighing less than $45 \mathrm{~kg}$ ) is adapted to allow rapid interfacing to electrospray ionization (ESI), desorption electrospray ionization (DESI), and direct analysis in real time (DART). Data from analyses of drugs (including metamphetamine, cocaine, and heroin), food (cinnamon, peppermint, and spearmint), and explosives (RDX and PETN) are presented with signal levels that are comparable to those of laboratory (benchtop) instruments.

Two papers from researchers at Brigham Young University use toroidal ion trap designs. Lee and colleagues use a GC coupled to an ion trap in a miniature system $(13 \mathrm{~kg})$ with an $\mathrm{m} / \mathrm{z}$ range from 50 to 500 with better than unit resolution for an ion of $m / z=222$. Mass spectra of GB and VX nerve agents are presented. Austin et al. present design and simulation of a novel planar ion trap in which the electrodes consist of two ceramic disks lithographically imprinted with concentric metal rings overlaid with resistive material. The novelty here is that the arrangement can produce any 
trapping field geometry including toroidal and traditional Paul-trap, but on a smaller scale.

The final paper in this issue reports on arguably the smallest complete mass spectrometer system to date. Yang and colleagues at the Analytical Instrumentation Research Institute, Korea, demonstrated a complete battery-powered, palm-portable mass spectrometer (PPMS) system including an ion trap, electronics, display, and ion-getter pump vacuum system weighing a mere $1.48 \mathrm{~kg}$. Spectra showing detection of organic gases (toluene to $6 \mathrm{ppm}$ and DMMP to $22 \mathrm{ppm}$ ) diluted in air are demonstrated.

Taken together, the contributions in this special focus highlight the enormous information-rich capabilities that mass spectrometry offers in harsh environments and field conditions. There is no doubt that ongoing developments in this area will see the emergence of portable instruments with improved characteristics, leading to further exciting applications. The 7 th HEMS workshop is scheduled for the fall of 2009; all those interested are most welcome either as attendees or better still as participants (www.hems-workshop.org).

Stephen Taylor

6th HEMS Workshop and Guest Editor, JASMS

Department of Electrical Engineering and Electronics University of Liverpool

Liverpool, UK

Veronica M. Bierbaum Associate Editor, JASMS

Department of Chemistry and Biochemistry University of Colorado Boulder, Colorado, USA 